\title{
Backtracking-free Interactive Music Scores with Temporal Relations over Rythms
}

\author{
Mauricio Toro \\ Universidad Eafit
}

October 4, 2018

\section{Introduction}

Constraint programming is a declarative programming paradigm wherein relations between variables are stated in the form of constraints. Applying constraint programming to classical music is a very natural idea because harmonization rules are easily stated. This is also true for many contemporary music. The reader may see, for instance, Situation, a constraint solving library that has been used in several compositions [18].

An interesting kind of musical problems solved with constraint programming are those concerining rhythmical structures. As an example, to find $n$ rhythmical patterns of fixed lengths, each pattern played on one voice, such that two different voices never play the beginning of a note simultaneaously. Such problem was proposed by composer Mauro Lanza and it has been extensively studied by Truchet et al., among other music problems where a constraint programming was found to be very pertinent [38].

Constraint satisfaction problems (CSPS) are mathematical problems defined as a set of objects whose state must satisfy a number of constraints or limitations. Formally, a CSP is defined as a triple $\langle X, D, C\rangle$, where $X$ is a set of variables, $D$ is a domain of values, and $C$ is a set of constraints. A solution is an evaluation of the variables that satisfies all constraints with values from their domains. CSPs can be solved with complete methods such as forward checking, used by Situation, but they are NP-complete problems in general.

Another alternative to solve CSPs is to use (incomplete) local search algorithms to find interatively an approximative solution. Local search is a metaheuristic for solving computationally hard optimization problems. An example of local search is adaptive search: A computational method that optimizes a problem by iteratively trying to improve a candidate solution with regard to the problem constraints (and possibly an optimization function). Truchet et al. found appropriate this method for multiple problems in music composition [38].

In spite of the advances in constraint solving for music compositions problems, as far as we know, there is little research on solving CSPs during real-time music 
interaction. In this paper we will focus on interactive scores, a formalism for the design of interactive scenarios closely related to constraint programming. Interactive scores has been a subject of study since the beginning of the century [9]. A recent model [3] has inspired two applications: iScore to compose and perform Electroacoustic [1] music and Virage to control live spectacles and for interactive museums [2].

Scenarios in interactive scores are represented by temporal objects, temporal relations and interactive events. Examples of temporal objects are sounds, videos and light controls. Temporal objects can be triggered by interactive events: The user can choose a date for the start or end of a temporal object. In addition, several temporal objects can be active simultaneously. A temporal object can contain other temporal objects: this hierarchy allows us to control the start or end of a temporal object by controlling the start or end of its parent. Temporal relations provide a partial order for the execution of the temporal objects; for instance, temporal relations can be used to express precedence between two objects.

All temporal and hierarchical relations can be easily stated by the means of constraints. In fact, there is a CSP associated to an interactive score. In such problem, the variables are the starting and ending dates of the temporal objects, the domains are finite for practical applications, and the constraints are the temporal and hierarchical relations of the problem. We will formally present such CSP and the formal definition of interactive scores in section 2. Later, in section 3, we will show that under certain conditions, such CSP can be solved in polynomial time.

Obviously, we do not have a polynomial algorythm to solve all instances of the problem. Consider, for instance, the case where the durations of the temporal objects are not singles values nor intervals, but discrete values. Durations represented as sets of discrete values are useful to model rythmical problems as the one presented in Figure 1. They are also useful when modeling loops and conditional branching on the lines of [29]. Nonetheless, the problem of finding if there is a solution for the CSP associated to an interactive score, in the general case, is NPComplete by encoding from the subset sum problem, as we will describe in section 3.

Process calculi has been applied to the modeling of interactive music systems $[27,26,32,37,25,36,4,33,28,12,23,20,22,24,5,31,21,29,30,19]$ and ecological systems [34, 14, 35, 15]. In addition, research on algorithms [13, 11, 17] and software engineering [16] also contributes to this field.

\subsection{Related work}

Satisfaction (SAT) is the problem of determining if the variables of a given Boolean formula can be assigned in such a way as to make the formula evaluate to true. Many CSPS can be easily translated into SAT instances. A class of algorithms called SAT solvers can efficiently solve a large enough subset of SAT instances to be useful in various practical areas; however, no current such methods can efficiently solve all SAT instances. 


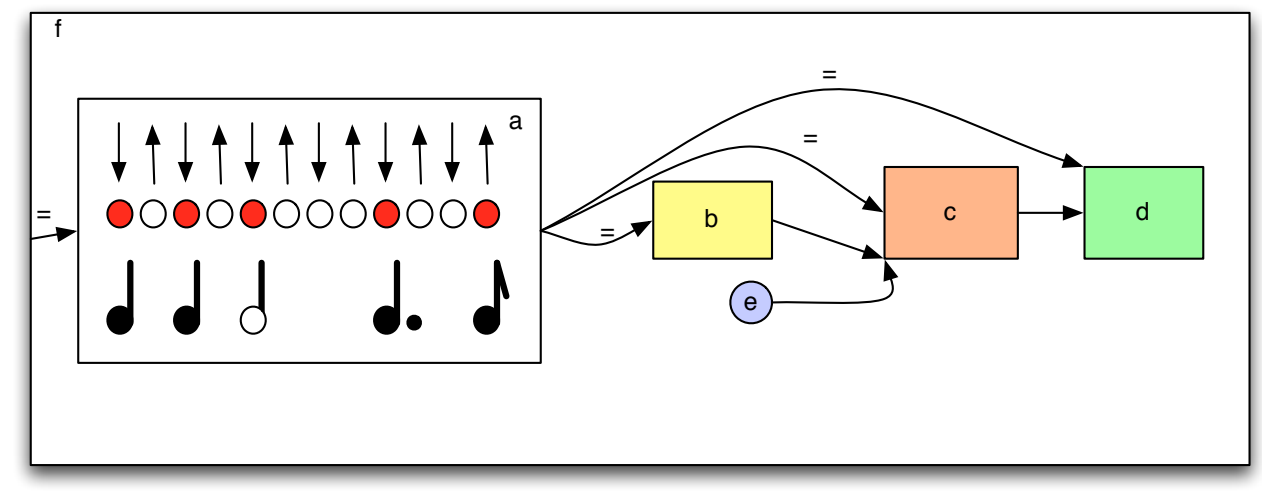

Figure 1: In the score above, temporal objects $b, c$ and $d$ are preceeded by $a$. The possible durations of $a$ are given by a rythmic pattern that can be representd by the set $[0,2,4,8,11]$, where each a eighth note lasts one discrete time-unit. Since $b, c, d$ are preceeded by $a$, they have to be executed zero, two, four, eight or 11 time units after $f$ starts.

We believe that none of constraint solving techniques described above nor SAT solvers are appropriate to compute the satisfaciblity of an interactive score during a real-time performance. (1) Forward checking has exponential time and space complexities. (2) The iterative nature of adaptive search will cause the starting and ending times of the objects to fluctuate along time and may cause inconsistencies with values already chosen in the past. In addition, local search does not guarantee a solution, for instance, it may cause that two temporal objects that are supposed to finish at the same time finish within a noticeable interval of time, which is unacceptable in most compositions. (3) Finally, we conjecture that SAT solvers are not appropriate for real-time interaction: they will not allow to interact the computer with an user without noticeable delays.

We believe that using space-efficient backtrack-free representation of constraint satisfaction problems [6] is appropriate for real-time interaction. This approach has never been used in real applications as far as we know altough the results with random generated CSPs are quite promising. Such representation consists on removing values from the domain of the variables that lead to dead-ends, trading the need of backtracking by a preprocessing of the problem. The algorithm is parametric on a seed: a solution that is already known and must be preserved after reducing the domains.

A particular case of these algorithms is 1-BFR, which guarantees a spaceefficient representation but also solution lost. This algorithm sadds notequals constraints $(\neq)$ to the original problem, which is equivalent to remove values from the domains. The 1-BFR algorithm is presented in section 4 . A generalisation of 1-BFR is $n$-BRF, where $n$ is the number of variables. This algorithm adds $n$-ary constraints 
and guarantees no solution lost but space requirements may be exponential in the size of the problem, thus is not of practical use for interactive scores.

\subsection{Contribution}

In this paper we propose an extension of the 1-BFR algorithm proposed in [6] to allow multiple seeds. Since the 1-BFR requires a known variable ordering, we propose a static variable ordering for the CSPs of a subclass of interactive scores. We also present results on the complexity of those CSPs to indentify the tractable (polynomially solvable) cases and the cases where the 1-BFR algorithm is of practical use in section 3 . We show the pertinence of 1-BFR in a score whose temporal objects' durations are represented as sets of pair numbers in section 4 . Finally, we discuss results, conclusions and future work in section 5 .

\section{Interactive Scores}

Interactive scores are composed by temporal relations and temporal objects. A temporal object has two points identifiers: for its start and for its end. In the implementation, points have an external action associated (e.g., turn on the lights or play a sound).

Let $\Sigma$ be set of names, $P$ a set of all point identifiers, and let $\mathscr{H}=(V, E)$, $V \subseteq \Sigma, E \subseteq V \times V$, be a directed tree called the object hierarchy. Temporal objects must have a unique object identifier to guarantee that the hierarchy is always a tree. Interactive points are a special case of temporal objects with zero-duration. Interactive points must be temporal objects instead of point identifiers (ids). Consequently, point ids are either the start or the end of an object. Interactive points control the starting or ending time of so-called static temporal objects.

Definition 2.1 (Temporal object (TO). ). Let $\mathscr{H}=(V, E)$ an object hierarchy and $P$ the set of point ids. A TO is a tuple $o_{\mathscr{H}}=\langle s p, e p, \Delta, v\rangle$, where sp,ep $\in P$ are the (unique) start and end point ids, $\Delta \subseteq \mathbb{N}$ are the possible durations, $v \in V$ is the object identifier. We assume an injective function object $\mathscr{H}: V \rightarrow \mathscr{T}$ giving, for each name, the corresponding TO. The set of all TOs is $\mathscr{T}$. An interactive point is an element of $\mathscr{T}$ such that $\Delta=\{0\}$. Functions $\operatorname{sp}(v), \operatorname{ep}(v)$ and $\mathrm{d}(v)$ return, respectively, start point, end point and the durations of $\operatorname{object}_{\mathscr{H}}(v)$.

A partial order among temporal objects is given by point-to-point relations without disjunction nor inequality: Qualitative temporal relations between the start or end of two temporal objects; for instance, the start of $a$ is executed before the end of $b$, or the start of $a$ is executed at the same time than the start of $b$ [7].

Definition 2.2 (Temporal Relation (TR). ). A TR is a tuple $\langle p$, rel, $q\rangle$, where $p, q \in$ $P$ are two different point ids of two different TOs, rel $\in \operatorname{Rel}=\{\langle\rangle,, \leq, \geq,=\}$. The set of all TRs is $\mathscr{E}$. 


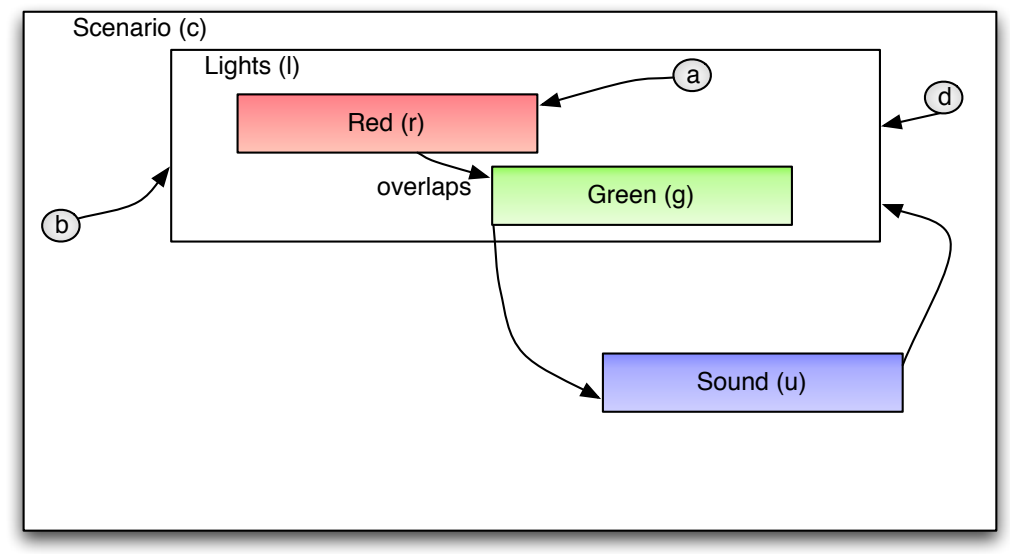

Figure 2: Example of a score. The duration of all temporal objects are pair numbers between 1 and 10 .

A score is a tuple consisting of an object hierarchy, a set of temporal relations over those objects and a set of point identifiers, such that two interactive points cannot start at the same time, for simplicity. Figure 4.4 is a example of a score.

Definition 2.3 (Interactive Score. ). An interactive score is a tuple $\langle\mathscr{H}, R, P\rangle$, where $\mathscr{H}=(V, E)$ is an object hierarchy, $P$ is the set of point identifiers, and $R \subseteq(P \times \operatorname{Rel} \times P)$ is given by $\langle p, r, q\rangle \in R$ iff $p$ is the start or end point of an object, $q$ is the start or end point of another object, and they are related by a point-to-point relation $r$. $R$ must be such that two interactive points in $V$ do not start at the same time. Let $\mathscr{I}$ be the set of all interactive scores.

\subsection{Temporal Constraints of the Score}

The temporal specification of a score is the conjunction of constraints over the start and end times of the temporal objects of the score. We use the following notation for temporal constraints: $t+\Delta={ }^{d e f}\left\{t^{\prime} \mid t^{\prime}=t+\delta, \delta \in \Delta\right\}$. Function ct $: P \rightarrow \mathbb{N}$ is a bijective function that associates a point identifier to the a variable that represents the time when it is launched.

Definition 2.4 (Temporal Specification of an Interactive Score. ). The temporal specification $\operatorname{ts}(s)$ of an interactive score $s=\langle\mathscr{H}, R, P\rangle, \mathscr{H}=(V, E)$, is the conjunction of constraints:

1. For each $a \in V, \operatorname{ct}(\operatorname{ep}(a)) \in \operatorname{ct}(\operatorname{sp}(a))+\mathrm{d}(a)$, where $\mathrm{d}(a)$ are the durations of $a$.

For each node $x$ in the subtree rooted at $a, \operatorname{ct}(\operatorname{sp}(x)) \geq \operatorname{ct}(\operatorname{sp}(a))$ and $\operatorname{ct}(\operatorname{ep}(a)) \geq$ $\operatorname{ct}(\mathrm{ep}(x))$. 


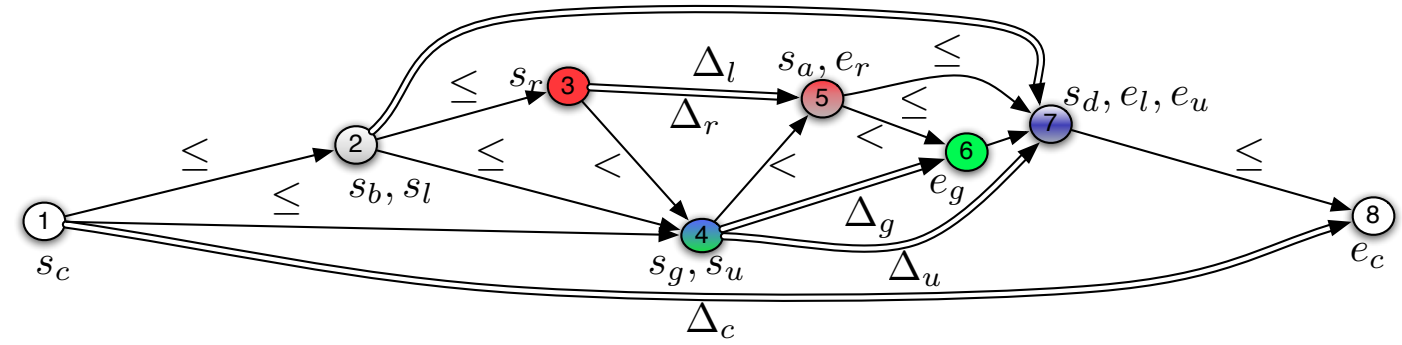

Figure 3: Temporal Constraints of the Score. For simplicity, two points that must happen at the same time are represented with a single variable and rebundant relations induced by the hierarchy are removed. A constraint $\Delta$ between a variable $x$ and a variable $y$ means that $y \in x+\Delta$.

\section{For each $\langle p, r, b\rangle \in R$, $\operatorname{ct}(p) r \operatorname{ct}(q)$, where $r \in\{=,<,>, \leq, \geq\} 1$ |}

The playability of a score can be decided by solving a constraint satisfaction problem (CSP). This property states that all temporal objects will be played during execution; this is desirable because a score can be over-constrained and therefore not playable.

The CSP associated to an interactive score is given by: a variable $x_{i}$ for starting, ending or interactive point in the score; the domain $\mathbb{N}_{\mathbb{F}} \cup\{\infty\}$ for each variable, where $\mathbb{N}_{\mathbb{F}}$ is a finite subset of $\mathbb{N}$; and the the single constraint tc $(\varepsilon)$. As an example, the constraint graph for the score in Figure 4.4 is presented in Figure 3

\section{Time Complexity of the Playbility of a Scores}

In what follows we will show that that the problem is NP-complete in the general case, but there is an interesting subclass that is tractable.

\subsection{The NP-complete case}

We will show that the decision problem of the subset sum can be encoded as an interactive score. The problem is stated as follows: Given a set of weights $\left(\left\{w_{1}, w_{2} \ldots w_{n}\right\}\right)$ of $n$ objects and an integer $W$, does any non-empty subset sum to $W$ ? Note that Subset sum can also be thought of as a special case of the knapsack problem.

- $\sum_{i=1}^{n} w_{i} \cdot x_{i}=W, x_{i} \in\{0,1\}$, where at least one $x_{i} \neq 0$.

\footnotetext{
${ }^{1} r \in\{=,<,>, \leq, \geq\}$ has the usual interpretation over $\mathbb{N}$.
} 
There are several algorithms to solve subset sum, all with exponential time complexity in $n$, the number of objects. The most nave algorithm would be to cycle through all subsets of $k$ numbers and, for every one of them, check if the subset sums to the right number. The running time is of order $\mathrm{O}\left(n 2^{n}\right)$, since there are $2^{n}$ subsets and, to check each subset, we need to sum at most $n$ elements. The best algorithm known runs in time $\mathrm{O}\left(2^{n / 2}\right)$ [10].

In what follow we present the temporal constraints of the score presented in Figure 4 For $1 \leq i \leq n$,

- $e_{i} \in s_{i}+\left\{0, w_{i}\right\}$

- $e_{s}=s_{s}+W$

- $e_{i}=e_{s}$

- $s_{s}=s_{i}$

The reader can easily verify that these constraints are equivalent to the constraint

- $\sum_{i=1}^{n} w_{i} \cdot x_{i}=W, x_{i} \in\{0,1\}$.

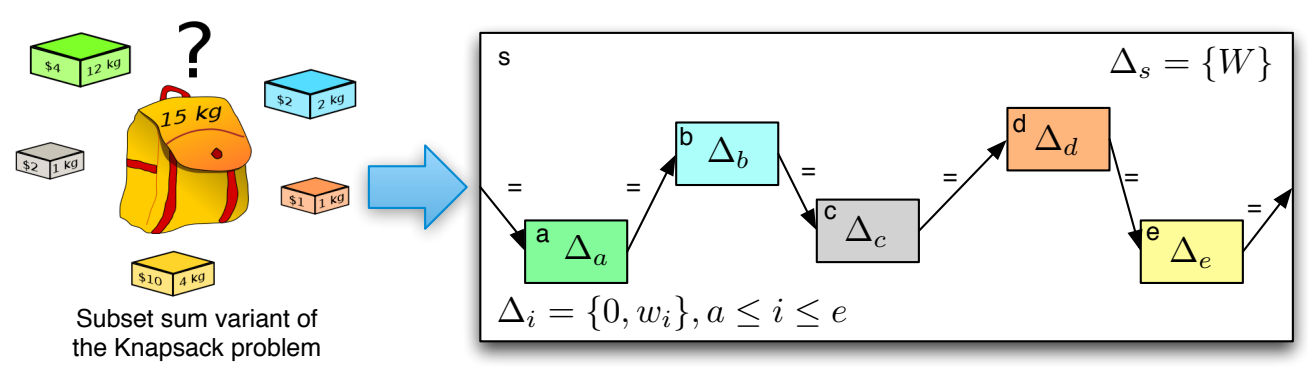

Figure 4: Encoding of the subset sum problem into an Interactive Score. Note that the subset sum problem is a variant of the knapsack decision problem where costs are not taken into acount and the goal is to find if there is a subset of the elements that fills exactly the knapsack capacity.

It is trivial to show that there is a polynomial algorithm to check whether a solution satisfies the problem. Such algorithm replaces the values of $x_{i}$ from the solution in the equation above. In conclusion, the subset sum can be encoded as the playbility (i.e., satisfaciblity) of an interactive score and there is a polynomial time algorithm to check if the solution is true, thus the satisfacibility of an interactive score is NP-complete.

\footnotetext{
${ }^{2}$ Knapsack picture taken from the Wikipedia.
} 


\subsection{The tractable subclass}

If durations are intervals of integers without holes, the playbility can be reduced to compute the path of minimum weight in a graph. Given that there are substractions to represent the duration of the temporal objects, there will be some negative coeficients. Dijkstra's algorithm is not appropriate because there are negative coefficients. Therefore, a simple solution is to use Floyd-Warshall algorithm, which complexity is $O\left(n^{3}\right)$, where $n$ is the number of points (starting, end and interactive points) in the score.

This problem is equivalent to the well-known Simple Temporal Problem (STP) [8]. A STP consists on a set of point variables $X=\left\{x_{1}, \ldots x_{n}\right\}$ and binary constraints over those variables $C=\left\{c_{1}, \ldots, c_{n}\right\}$ of the form $c_{k}: x_{i}-x_{j} \in[a, b]$ with $a, b \in \mathbb{N} \cup \infty$. There are better algorithms than Floyd-Warshall for this problem, for instance, directed path consistency (DPC) [8] and partial path consistency (PPC). The complexity of DPC is $O\left(n W * d^{2}\right)$, where $W * d$ is the induced lenght of the graph along a given ordering $d$.

Arc-consistency $(A C)$ is not equivalent to satisfacibility in this problem because the constraint graph usually constains cycles, thus we cannot recursively propagate values without the need of bactracking. Nonetheless, we will discuss in section 4 that there exists a static variable ordering for the CSPs of this subclass of interactive scores that allows us to recursively propagate values using arc-consistency, even in presence of cycles, without the need of backtrack. Complexity of arc-consistency is $\mathrm{O}\left(e d^{2}\right)$ for $\mathrm{AC}-7$, where $e$ is the number of arcs and $d$ the maximum domain among the variables. Conjecture: Its performance is usually better than DPC ?

A recent option is "Incrementally Solving STNs by Enforcing Partial Path Consistency". Discuss this in the conclusions.

\section{Backtrack-free Interactive Scores}

In this section we present the original seeded algorithm to find a 1-BFR form of a CSP. We also present our extension of such algorithm using multiple seeds, and finally, we show an example of how to use 1-BFR algorithms in the CSP of an interactive score.

\subsection{The seeded algorithm}

It was introduced in [6] an algorithm to find a 1-BFR form of a CSP that guarantees that a known solution of the problem (a seed) will be part of the solutions of the 1BFR form of the CSP. Its space complexity is polynomial in the number of variable because it only needs to represent the domains of each variable.

What is $P_{i}$ ?

What is $P P_{i}$ ?

How does the algorithm works... 
Its correctness was also proved in [6]. The argument is as follows. The only place where the algorithm could fail is in line 8 if it is impossible to choose $a$ such that $a \notin s^{*}$. ?? ARGUMENT THIS. A failfure in line 10 enforcing arc-consistency is neither possible. To see that this cannot happen, consider that we never remove a value that is the seed, thus there is always at least one value that can be chosen in the domain of each variable.

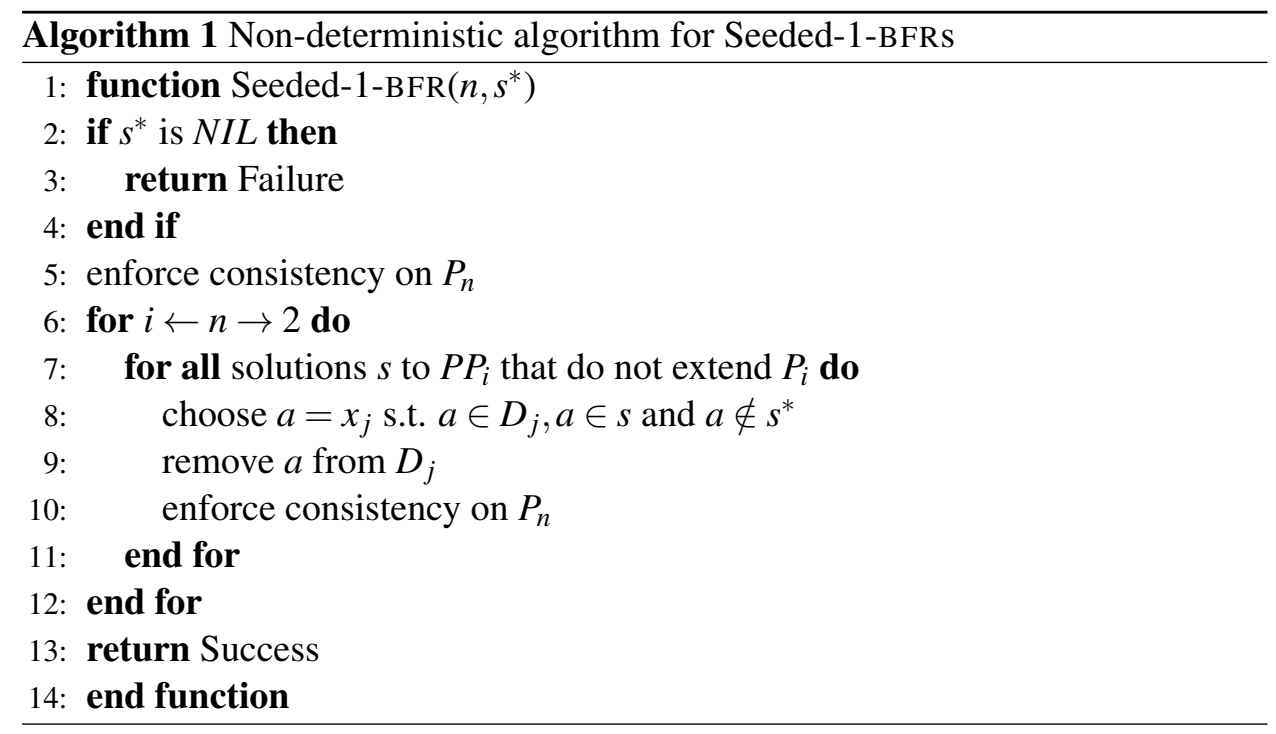

\subsection{Multi-seeded Algorithm}

A simple extension to the 1-seeded algorithm into a multi-seeded algorithm. We present a deterministic version of the algorithm, which closer to the implementation than the 1-seeded algorithm presented in [6]. Function MultiSeeded-1BFR enforces consistency on $\mathscr{P}_{n}$ and delegates the rest of the computation to MultiSeeded-1-BFR-sons that recursively reduces a value from the domain from each solution of $\mathscr{P}_{i}$ that do not extend $\mathscr{P} \mathscr{P}_{i}$. This function delegates the traversal of each solution that do not extend $\mathscr{P} \mathscr{P}_{i}$ to MultiSeeded-1-BFR-brothers.

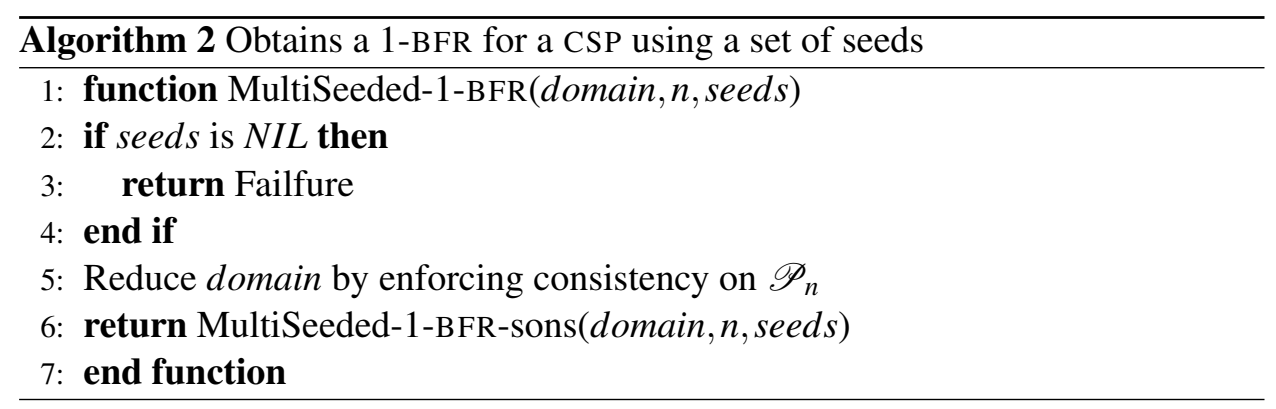



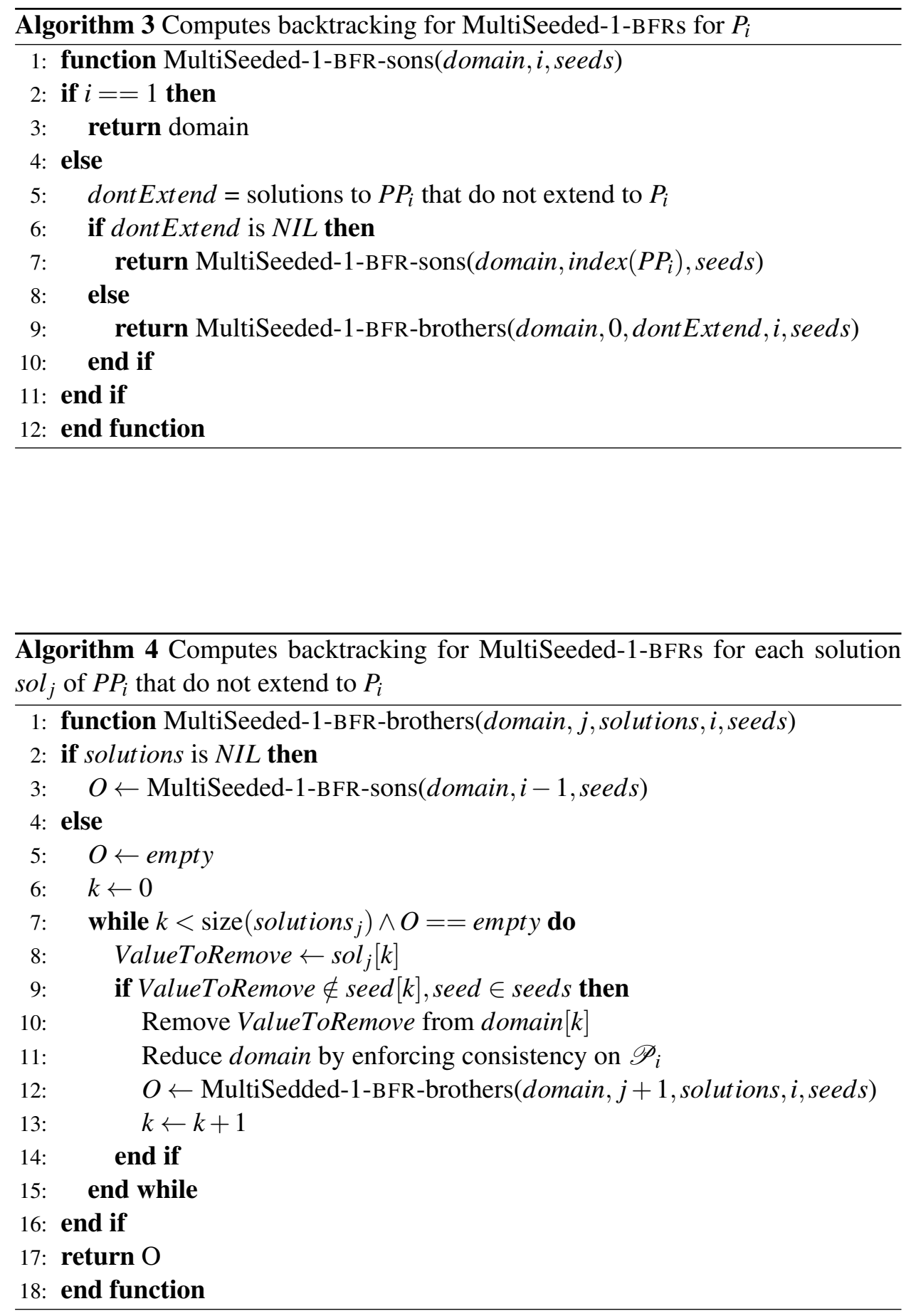


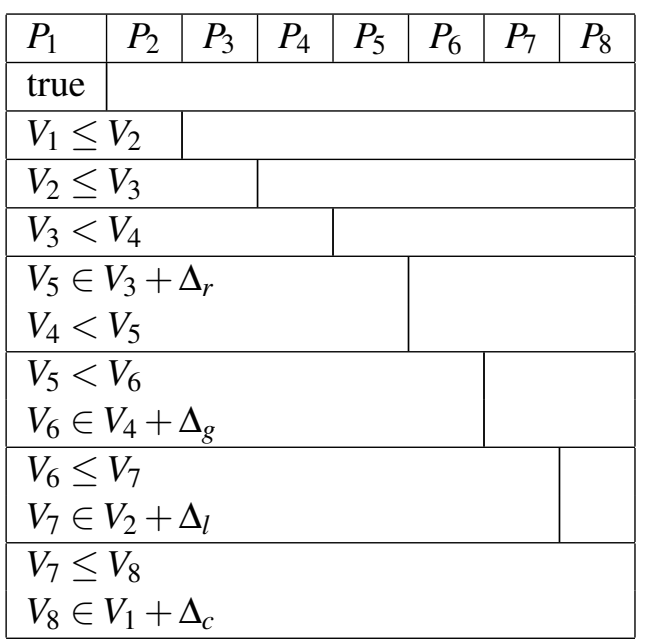

Table 1: Subproblems for the CSP of the interactive score of Figure 4.4, whose constraint graph is presented in Figure 3. Since there is a total order, the parent of problem $i$ is problem $i-1$.

\subsection{Variable ordering for Interactive Scores}

Variable ordering in Music is important and ... [?, ?]

For the CSP of a score, we must choose a variable ordering such that respects the partial order stablished by the temporal relations and the hierarchy.

Proposition 4.1. When the durations are intervals of integers and the variable ordering respects the partial order given by the temporal constraints of the score, then the problem after enforcing Arc Consistency is in BFR form for any variable ordering that respects the partial order given by $t c(s)$.

Proof. ?

\subsection{Study case}

In what follow we study the 1-BFR form of the score in Figure, provided that all the durations must be pair numbers between one and 10 . The temporal constraints of the score are presented in Figure 3. The variable ordering is such that it respects the order imposed by the temporal relations and the hierarchy, in this case we have a total order.

Table 1 presents the subproblems $P_{i}$ for the CSP associated to the score in Figure 4.4. Table ?? presents the results of enforcing arc-consistency on $P_{n}$. In the next section we present the results of executing the seeded and multiseeded algorithms for this problem. 


\section{Results and Conclusions}

\subsection{Summary}

\section{Introduction}

\section{Complexity}

\section{BFR}

Results For non-seeded we got just one solution and preliminary studies in [6] showed that it is slower. For the seeded case: Seed $\mathrm{i}=130$, namely $[0,0,1,3,5,7,8,10]$, we got 150/603 solutions. With one seed the maximum is $150 / 603$ solutions. With a 2-seeded approach using $\mathrm{i}=130$ and $\mathrm{j}=306$, namely $[0,2,2,5,6,7,10,10]$, we got 190/603 solutions. Using 3-seeded only made the problem slowler and did not ?? more solutions.

\subsection{Conclusions}

\section{Multiseeded works better than single seeded, but it's slower}

Tractable disjunctions, ORD-horn, etc Allen without disjunction is tractable. Disjunction without equality and without ,?? is tractable. ord-horn is tractable. other kinds tractable over reals. most cases in integers and non tractable.

the n voices problem We will need global constraints or disjunctions of temporal objects. We cannot have a static order in this case. The bfr approach does not longer work.

\subsection{Future work}

We foresee two simple extensions to our approach, already described in [6]. Use Branch-and-Bound search to find the 1-BFR form that represents tha maximum number of solutions and use the inverse of Geelen's promise heuristic during search (i.e., choose the pair with the most conflicts with values in the domain of neighboring variables).

\section{References}

[1] A. Allombert, G. A. Assayag, and M. Desainte-Catherine. Iscore: a system for writing interaction. In Proc. of DIMEA '08, pages 360-367, New York, NY, USA, 2008. ACM. 
[2] A. Allombert, P. Baltazar, R. Marczak, M. Desainte-Catherine, and L. Garnier. Designing an interactive intermedia sequencer from users requirements and theoretical background. In Proc. of ICMC 2010, 2010.

[3] A. Allombert, M. Desainte-Catherine, J. Larralde, and G. Assayag. A system of interactive scores based on qualitative and quantitative temporal constraints. In Proc. of Artech 2008, Porto, Portugal, 2008. The Artech International Association.

[4] A. Allombert, M. Desainte-Catherine, and M. Toro. Modeling temporal constrains for a system of interactive score. In G. Assayag and C. Truchet, editors, Constraint Programming in Music, chapter 1, pages 1-23. Wiley, 2011.

[5] J. Aranda, G. Assayag, C. Olarte, J. A. Pérez, C. Rueda, M. Toro, and F. D. Valencia. An overview of FORCES: an INRIA project on declarative formalisms for emergent systems. In P. M. Hill and D. S. Warren, editors, Logic Programming, 25th International Conference, ICLP 2009, Pasadena, CA, USA, July 14-17, 2009. Proceedings, volume 5649 of Lecture Notes in Computer Science, pages 509-513. Springer, 2009.

[6] J. C. Beck, T. Carchrae, E. C. Freuder, and G. Ringwelski. A space-efficient backtrack-free representation for constraint satisfaction problems. International Journal on Artificial Intelligence Tools, 17(4):703-730, 2008.

[7] M. Broxvall and P. Jonsson. Point algebras for temporal reasoning: algorithms and complexity. Artif. Intell., 149(2):179-220, October 2003.

[8] R. Dechter, I. Meiri, and J. Pearl. Temporal constraint networks. Artif. Intell., 49(1-3):61-95, 1991.

[9] M. Desainte-Catherine and N. Brousse. Towards a specification of musical interactive pieces. In Proc. of CIM XIX, Firenze, Italy., 2003.

[10] S. Martello and P. Toth. Knapsack problems: algorithms and computer implementations. John Wiley \& Sons, Inc., New York, NY, USA, 1990.

[11] J. D. A. Moreno, S. Passos, and M. Toro. On-line assembling mitochondrial DNA from de novo transcriptome. CoRR, abs/1706.02828, 2017.

[12] C. Olarte, C. Rueda, G. Sarria, M. Toro, and F. Valencia. Concurrent Constraints Models of Music Interaction. In G. Assayag and C. Truchet, editors, Constraint Programming in Music, chapter 6, pages 133-153. Wiley, Hoboken, NJ, USA., 2011.

[13] C. Patiño-Forero, M. Agudelo-Toro, and M. Toro. Planning system for deliveries in Medellín. ArXiv e-prints, Nov. 2016. 
[14] A. Philippou and M. Toro. Process Ordering in a Process Calculus for Spatially-Explicit Ecological Models. In Proceedings of MOKMASD'13, LNCS 8368, pages 345-361. Springer, 2013.

[15] A. Philippou, M. Toro, and M. Antonaki. Simulation and Verification for a Process Calculus for Spatially-Explicit Ecological Models. Scientific Annals of Computer Science, 23(1):119-167, 2013.

[16] M. Raul, T. Mauricio, and C. Luz. Definicion de la arquitectura de referencia de un dominio: de la elucidacion al modelado. In R. Mazo, editor, Guia para la adopcion industrial de lineas de productos de software, pages 193-210. Editorial Eafit, 2018.

[17] J. M. C. Restrepo, A. F. Z. Palacio, and M. Toro. Assembling sequences of DNA using an on-line algorithm based on debruijn graphs. CoRR, abs/1705.05105, 2017.

[18] C. Rueda, M. Lindberg, M. Laurson, G. Block, and G. Assayag. Integrating constraint programming in visual musical composition languages. In ECAI 98 Workshop on Constraints for Artistic Applications, Brighton, 1998.

[19] M. Toro. Exploring the possibilities and limitations of concurrent programming for multimedia interaction and graphical representations to solve musical csp's. Technical Report 2008-3, Ircam, Paris.(FRANCE), 2008.

[20] M. Toro. Probabilistic Extension to the Factor Oracle Model for Music Improvisation. Master's thesis, Pontificia Universidad Javeriana Cali, Colombia, 2009.

[21] M. Toro. Towards a correct and efficient implementation of simulation and verification tools for probabilistic ntcc. Technical report, Pontificia Universidad Javeriana, May 2009.

[22] M. Toro. Structured interactive musical scores. In M. V. Hermenegildo and T. Schaub, editors, Technical Communications of the 26th International Conference on Logic Programming, ICLP 2010, July 16-19, 2010, Edinburgh, Scotland, UK, volume 7 of LIPIcs, pages 300-302. Schloss Dagstuhl - Leibniz-Zentrum fuer Informatik, 2010.

[23] M. Toro. Structured Interactive Scores: From a simple structural description of a multimedia scenario to a real-time capable implementation with formal semantics . PhD thesis, Univeristé de Bordeaux 1, France, 2012.

[24] M. Toro. Structured interactive music scores. CoRR, abs/1508.05559, 2015.

[25] M. Toro. Probabilistic Extension to the Concurrent Constraint Factor Oracle Model for Music Improvisation. ArXiv e-prints, Feb. 2016. 
[26] M. Toro. Probabilistic Extension to the Concurrent Constraint Factor Oracle Model for Music Improvisation . Inteligencia Artificial, 57(19):37-73, 2016.

[27] M. Toro. CURRENT TRENDS AND FUTURE RESEARCH DIRECTIONS FOR INTERACTIVE MUSIC. Journal of Theoretical and Applied Information Technology, 69(16):5569-5606, 2018.

[28] M. Toro, C. Agón, G. Assayag, and C. Rueda. Ntccrt: A concurrent constraint framework for real-time interaction. In Proc. of ICMC '09, Montreal, Canada, 2009.

[29] M. Toro and M. Desainte-Catherine. Concurrent constraint conditional branching interactive scores. In Proc. of SMC '10, Barcelona, Spain, 2010.

[30] M. Toro, M. Desainte-Catherine, and P. Baltazar. A model for interactive scores with temporal constraints and conditional branching. In Proc. of Journées d'Informatique Musical (JIM) '10, May 2010.

[31] M. Toro, M. Desainte-Catherine, and J. Castet. An extension of interactive scores for multimedia scenarios with temporal relations for micro and macro controls. In Proc. of Sound and Music Computing (SMC) '12, Copenhagen, Denmark, July 2012.

[32] M. TORO, M. DESAINTE-CATHERINE, and J. CASTET. An extension of interactive scores for multimedia scenarios with temporal relations for micro and macro controls. European Journal of Scientific Research, 137(4):396409, 2016.

[33] M. Toro, M. Desainte-Catherine, and C. Rueda. Formal semantics for interactive music scores: a framework to design, specify properties and execute interactive scenarios. Journal of Mathematics and Music, 8(1):93-112, 2014.

[34] M. Toro, A. Philippou, S. Arboleda, M. Puerta, and C. M. Vélez S. Meanfield semantics for a process calculus for spatially-explicit ecological models. In C. A. Muñoz and J. A. Pérez, editors, Proceedings of the Eleventh International Workshop on Developments in Computational Models, Cali, Colombia, October 28, 2015, volume 204 of Electronic Proceedings in Theoretical Computer Science, pages 79-94. Open Publishing Association, 2016.

[35] M. Toro, A. Philippou, C. Kassara, and S. Sfenthourakis. Synchronous parallel composition in a process calculus for ecological models. In G. Ciobanu and D. Méry, editors, Proceedings of the 11th International Colloquium on Theoretical Aspects of Computing - ICTAC 2014, Bucharest, Romania, September 17-19, volume 8687 of Lecture Notes in Computer Science, pages 424-441. Springer, 2014. 
[36] M. TORO, C. RUEDA, C. AGÓN, and G. ASSAYAG. Ntccrt: A concurrent constraint framework for soft real-time music interaction. Journal of Theoretical \& Applied Information Technology, 82(1), 2015.

[37] M. TORO, C. RUEDA, C. AGÓN, and G. ASSAYAG. Gelisp: A framework to represent musical constraint satisfaction problems and search strategies. Journal of Theoretical \& Applied Information Technology, 86(2), 2016.

[38] C. Truchet, G. Assayag, and P. Codognet. Omclouds, a heuristic solver for musical constraints. In MICO3, Metaheuristics International Conference, $\mathrm{Ky}-$ oto, 2003. 